\title{
The Technique of Multi-System Leak Detection for Spacecraft with Quadrupole Mass Spectrometer
}

\author{
R.X. YAN \\ Beijing Institute of Spacecraft Environment Engineering, Beijing, China
}

\begin{abstract}
As the development of spacecraft, the multi-system leak detection is needed. The multi-system leak detector on spacecraft by QMS (quadrupole mass spectrometer) has been developed and it has been used in the new spacecraft successfully. The problem of repeat and reliability of QMS in long time is avoided. The QMS calibration is solved by injecting the quantitative calibration gases into the cumulative vessel. In the atmosphere, the minimal $\mathrm{He}$ concentration can be test is $13 \times 10^{-9}$, CF4 concentration is $10 \times 10^{-9}$, Ne concentration is $3 \times 10^{-6}, \mathrm{SF}_{6}$ concentration is $0.3 \times 10^{-9}, \mathrm{Kr}$ concentration is $10 \times 10^{-9}$. For the collection chamber with $100 \mathrm{~m}^{3}$ volume, in 24 hours, the minimal He leak rate can be test is $1.5 \times 10^{-6} \mathrm{~Pa} \cdot \mathrm{m}^{3} / \mathrm{s}, \mathrm{CF}_{4}$ leak rate is $1.1 \times 10^{-6} \mathrm{~Pa} \cdot \mathrm{m}^{3} / \mathrm{s}$, Ne leak rate is $3.5 \times 10^{-5} \mathrm{~Pa} \cdot \mathrm{m}^{3} / \mathrm{s}, \mathrm{SF}_{6}$ leak rate is $3 \times 10^{-8} \mathrm{~Pa} \cdot \mathrm{m}^{3} / \mathrm{s}, \mathrm{Kr}$ leak rate is $1.1 \times 10^{-6}$ $\mathrm{Pa} \cdot \mathrm{m}^{3} / \mathrm{s}$.
\end{abstract}

KEYWORD: Mass spectrometer; Leak detection; Spacecraft; Quadrupole mass spectrometer

\section{INTRODUCTION}

Nowadays, helium mass spectrometer technology is widely used in the leak detection of spacecraft. This technology can effectively solve leak problem of single system. As the development of spacecraft, multi-system leak detection is needed. Based on the old detector, ESA invent a piezoelectricity crystal pulse sampling leak detector[1], which contrast the ion flow of detectable gas to the compared gas. This method reduces effect of instability of vacuum system and mass spectrometer, the sensibility and reliability are increased. On the same sensibility demand level, the cost decreases clearly. The America Sodal[2] research on the mass spectrometer using a pulsed gas sampling system, and acquire satisfying result. After large of experiments of detectable gas analysis machine, and based on the GAM500 on-line mass produced by Germany IPI, we developed alternate circle sampling system and get over the instability of long-time measuring[3-5]. The multi-system leak detector on spacecraft by mass spectrometer has been developed and it has been used in the new spacecraft successfully.

\section{COMPOSITION OF EQUIPMENT AND TEST THEORY}

\subsection{Composition of equipment}

The equipment is composed by GAM500 on-line mass spectrometer, gas cycle sampling system, collection chamber, charging equipment and so on, the theory chart is shown on Fig.1.

GAM500 on-line mass spectrometer is combined with QMA422 mass spectrometer, Ges070 sampling system, TMU0650 molecular pump vacuum system and 8 sampling valve, which can acquire more sampling points.

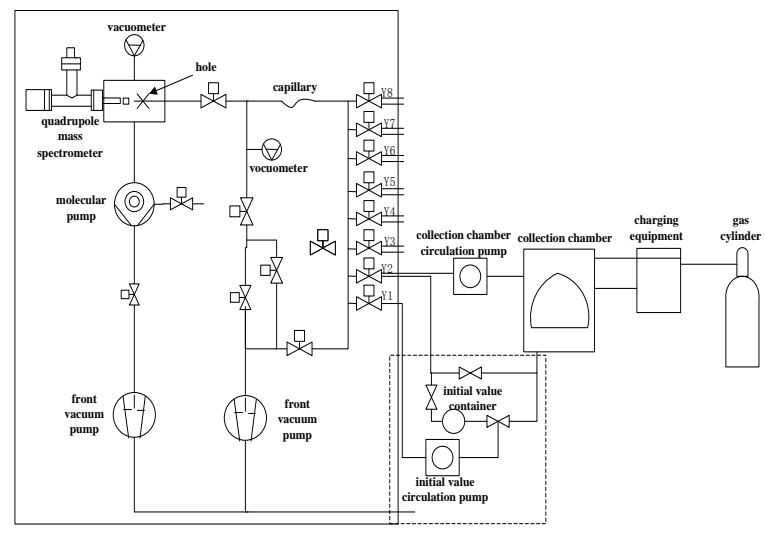

Fig.1 principle scheme of spacecraft multi-system leak detector by mass spectrometer

Gas cycle sampling system is combined with two route: one includes sampling valve, collection chamber cycle pump, stop valve, the other route includes initial value container, sampling valve, stop valve and initial value container cycle pump. 
A ventilator cycle system is installed in the collection chamber and the blast volume index is: circulate the air in collection chamber every 5 minutes. The airproof capability is: after charging $2 \mathrm{kPa}$ pure Nitrogen, the pressure reduces $150 \mathrm{~Pa}$ in 24 hours. Charging equipment is combined with compressor, gas cylinder, standard container, mechanical pump, control meter, control valve and so on. The charging equipment can extract the spacecraft system, charge the detectable gas, reclaim the detectable gas and infuse ration air to the collection chamber.

\subsection{Test theory}

Charge specific pressure detectable gas (there are some kinds of inert gases such as $\mathrm{He}, \mathrm{Ne}, \mathrm{Kr}, \mathrm{CF}_{4}$ in air) into different test systems in spacecraft, seal the inter-face and push into the collection chamber, open the fan in the collection chamber, reduce the detectable gas in the chamber. Close the chamber door and seal the chamber. Startup two cycle pump, close $K_{3}$, open $K_{1}$ and $K_{2}$ valves to make the initial value container air the same as chamber air. Open $\mathrm{K}_{3}$, close $\mathrm{K}_{1}$ and $\mathrm{K}_{2}$ valves, as the GAM500 operates well, cycle test collection chamber and the initial value container have the same detectable gas ion flow. Stay the spacecraft in the chamber for $t$ seconds to make the leak air accumulate and the density increases. Open the ventilator cycle system and initial value container cycle pump. The air in the initial value container and the air in the collection chamber flow into the mass spectrometer alternately, and we achieve corresponding ion flow $u_{i 10}$ and $u_{i 1}$. After testing several steady sampling cycle, charge standard gas $W_{i}\left(P_{i b} \cdot V_{b}\right)$, and then open the ventilator cycle system to make the standard gas, detectable air and air mixed uniformly. The ion flow in initial value container and chamber are $u_{i 20}$ and $u_{i 2}$ respectively. Because the test time is short and we test alternately, the stability of pumping speed, inspiration action and the stability of QMS can be ensured, and the test ion flow keep stable and can be repeated well. The ratio between standard flow $W_{i}$ and product of chamber volume and atmospheric pressure values around 1 percent. After charging standard gas, the whole pressure changes a little. We can acquire leak-rate of different spacecraft through this formula:

$$
Q_{i}=\frac{W_{i}\left(u_{i 1}-u_{i 10}\right)}{t\left[\left(u_{i 2}-u_{i 20}\right)-\left(u_{i 1}-u_{i 10}\right)\right]}
$$

In the equation, $W_{i}$ is the standard gas flow $\left(\mathrm{Pa} \cdot \mathrm{m}^{3}\right), W_{i}=P_{i b} \cdot V_{b}, V_{b}$ is the volume of standard gas flow container, $P_{i b}$ is the pressure of detectable air when flowing standard gas.

\section{PHYSICS ANALYSIS}

\subsection{Gas tightness effect}

In the multi-system leak detecting system on spacecraft by mass spectrometer, the collection chamber has a function to seal and accumulate detectable gas. Detectable gas leaking from collection chamber will influence the test dependability, so strict hermetic index is asked for.

The whole leak-rate test requires staying 24 hours in the collecting chamber. At this period of time, the environment temperature does not change, but keep stable between $23 \pm 5^{\circ} \mathrm{C}$, the air pressure at one same place are changeable between $\pm 500 \mathrm{~Pa}$. The air in the collection chamber can be regard as ideal air which satisfies the following formula:

$$
P V=\frac{M R T}{\mu}
$$

In the equation, $M$ is the mole mass $(\mathrm{kg} / \mathrm{mol}), P$ is the air pressure $(\mathrm{Pa}), \mu$ is the mass of air molecule $(\mathrm{kg}), V$ is the volume of collection chamber $\left(\mathrm{m}^{3}\right), R$ is the mole constant, $8.31 \mathrm{~J} /(\mathrm{mol} \cdot \mathrm{K}), T$ is the air temperature in collection chamber.

As the volume in collection chamber does not change, set the initial chamber pressure $P_{1}$, temperature $T_{1}$, air pressure $A_{1}$, the final chamber pressure after 24 hours $P_{2}$, temperature $T_{2}$, air pressure $A_{2}$. The pressure difference $\triangle P$ brought by temperature and air pressure changing turns out to be:

$$
\Delta P=\left(P_{1}+A_{1}\right)-\left(P_{2}+A_{2}\right) \frac{T_{1}}{T_{2}}
$$

Considering the air pressure and temperature changing in the chamber, we can obtain the pressure difference $2 \mathrm{kPa}$. When the spacecraft leaks and the chamber's tightness is low, detectable gases will leak from chamber with air and the density of detectable gases will reduce. If the leak of chamber is about $0.1 \%$, the effect to sensitivity and reliability would be small. Considering maximum pressure the chamber can support, the chamber can satisfy Nitrogen pressure reducing less than $150 \mathrm{~Pa}$, when the chamber suffers $2 \mathrm{kPa}$ pressure, which ensure the sensitivity and reliability.

\subsection{The effect analysis of cycle blast volume to leak detecting sensitivity}

For the multi-system leak detecting system on spacecraft by mass spectrometer, the detectable gases leak from the spacecraft have been mixed well with air in collection chamber so that the sampling gas can express the reality of spacecraft leak. The maximum volume of spacecraft leak detection collection chamber is $100 \mathrm{~m}^{3}$, in that case, if the gas in the chamber can cycle three times in 30 minutes, 
the gas would be mixed well. So the blast volume of cycle system should be bigger than $10 \mathrm{~m}^{3} / \mathrm{min}$.

\subsection{The pressure of mass spectrometer and ion flow variation}

The principle of multi-system leak detecting system by mass spectrometer can be equivalent as Fig.2(a). Set the volume of mass spectrometer chamber $V$, flow velocity of pump to mass spectrometer $S_{m}$, the diameter of sampling hole $A$, and the sampling is cycle type. The sampling periodicity is shown in Fig2(b), at the time $t_{a}$, flow in the sampling hole detectable gas which has base pressure $P_{0 i}$ and increment pressure $P_{i}$, and at the time $t_{b}$, the detectable gas has a base pressure $P_{0 i}$.
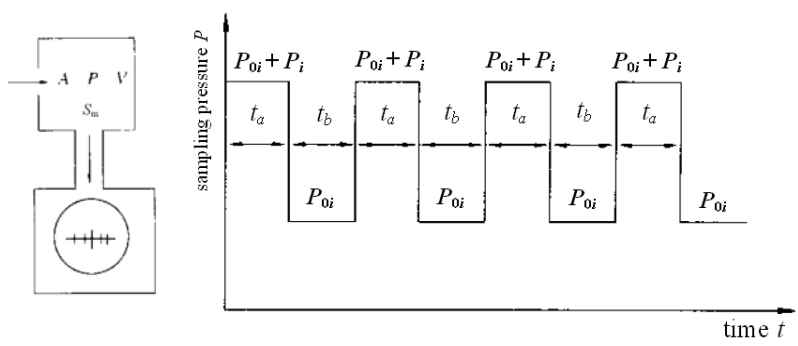

(a) principle scheme (b) sampling cycle principle scheme

Fig. 2 principle scheme of spacecraft multi-system leak detector by mass spectrometer sampling

At molecular flow state, the molecular number flow through the sampling hole to the spectrometer is $n v A / 4$. Because the detectable gas pressure in spectrometer is smaller than in the sampling pipeline, we can ignore the molecule flow from spectrometer to pipeline, which means that the gas flow from pipeline to the spectrometer only. At the sampling time $0 \sim t_{a}$, the detectable gas pressure in spectrometer accord with the following formula:

$$
V \frac{\mathrm{d} P}{\mathrm{~d} t}=\frac{1}{4} n v A R T-\left.P S_{m} \quad P\right|_{t=0}=P_{0 i}
$$

In the above expression, $v$ is the average molecule velocity of detectable gas, $\mathrm{n}$ is the gas density before flow into the spectrometer. To resolve equation (4), the sampling time is between $0 \sim t_{a}$, the pressure of spectrometer accord with formula (5).

$$
P=\frac{v A\left(P_{i}+P_{0 i}\right)}{4 S_{m}}+\left(P_{0 i}-\frac{v A\left(P_{i}+P_{0 i}\right)}{4 S_{m}}\right) \exp \left(-\frac{S_{m}}{V} t\right)
$$

The detectable gas flow through the alternating cycle sampling system and after a period of time $t_{a}$, the detectable gas pressure in spectrometer is:

$$
P_{a}=\frac{v A\left(P_{i}+P_{0 i}\right)}{4 S_{m}}+\left(P_{0 i}-\frac{v A\left(P_{i}+P_{0 i}\right)}{4 S_{m}}\right) \exp \left(-\frac{S_{m}}{V} t_{a}\right)
$$

After a period of time $t_{a}$, between time $t_{a} \sim t_{a}+t_{b}$, transform the initial value container gas, when detectable gas $P_{0 i}$ flow into the spectrometer, the corresponding formula is:

$$
\begin{aligned}
& V \frac{\mathrm{d} P}{\mathrm{~d} t}=\frac{1}{4} n v A R T-\left.P S_{m} \quad P\right|_{t=0}=P_{a} \\
& n=\frac{P_{0 i}}{R T}
\end{aligned}
$$

To solve the expression (7), we have:

$$
\begin{aligned}
& P=\frac{v A P_{0 i}}{4 S_{m}}+\left(P_{a}-\frac{v A P_{0 i}}{4 S_{m}}\right) \exp \left(-\frac{S_{m}}{V} t\right) \\
& P_{(a+b)}=\frac{v A P_{0 i}}{4 S_{m}}+\left(P_{a}-\frac{v A P_{0 i}}{4 S_{m}}\right) \exp \left[-\frac{S_{m}}{V}\left(t_{a}+t_{b}\right)\right]
\end{aligned}
$$

The volume of mass spectrometer chamber is $1 \mathrm{~L}$, the pumping speed is $60 \mathrm{~L} / \mathrm{s}, S_{m} / V=60 \mathrm{~s}^{-1}$, a cycle time $t_{a}$ and $t_{b}$ is in minute dimension, $S_{m} t_{a} / V$ and $S_{m} t_{b} / V$ are in 1000 dimension, the exponential item in expression (6) and (10) can be ignored and turned out to be:

$$
\begin{gathered}
P_{a}=\frac{v A\left(P_{0 i}+P_{i}\right)}{4 S_{m}} \\
P_{(a+b)}=\frac{v A P_{0 i}}{4 S_{m}}
\end{gathered}
$$

For the detectable gas in collection chamber which has a entrance pressure $\left(P_{0 i}+P_{i}\right)$ and the detectable gas in initial value container which has a inherent pressure $P_{0 i}$, with mass spectrometer which has emission current $I_{e}$ and sensitivity $S_{i}$, the ionic flow are $u_{i 1}$ and $u_{i 2}$ separately:

$$
u_{i 1}=\frac{I_{e} S_{i} A v\left(P_{0 i}+P_{i}\right)}{4 S_{m}} \quad u_{i 10}=\frac{I_{e} S_{i} A v P_{0 i}}{4 S_{m}}
$$

The ionic flow difference between detectable gas and the inherent detectable gas, which is the ionic flow of spacecraft leak can be shown as:

$$
u_{i 1}-u_{i 10}=\frac{I_{e} S_{i} A v P_{i}}{4 S_{m}}
$$

Put a standard gas sample $W_{i}\left(P_{i b} \cdot V_{b}\right)$ in the collection chamber which has available volume $V_{0}$, the detectable gas $P_{i 2}$ in the collection chamber has a increment $\Delta P_{i 2}$. We can also achieve the difference between detectable gas ionic flow which is mixed well with sample $u_{i 2}$ and inherent detectable gas ionic flow $u_{i 20}$ :

$$
u_{i 2}-u_{i 20}=\frac{I_{e} S_{i} A v\left(\Delta P_{i 2}+P_{i}\right)}{4 S_{m}}
$$

From formula (1) we can acquire that

$$
Q_{i}=\frac{W_{i} P_{i}}{t \Delta P_{i 2}}
$$

We can conclude from expression (14) that for the detectable specific pressure $P_{i}$, high sensitivity asks for bigger ion flow which can obtain by 
increasing the hole diameter. But increasing the hole diameter means more air would flow into the mass spectrometer, according to expression (12), to ensure the QMS effectively test to the detectable gas $i$, the pressure of mass spectrometer P should satisfy:

$$
\frac{v A P_{\text {air }}}{4 S_{m}} \leq P_{m}
$$

In the expression, $P_{\text {air }}$ is the absolute pressure of air, and $P_{m}$ is the upper limit of spectrometer.

At the same time, the ion flow of detectable gas is twice bigger than of noise ion flow, as the expression shows:

$$
2 u_{n i} \leq \frac{I_{e} S_{i} A v P_{i}}{4 S_{m}} \quad 2 u_{n i} \leq \frac{I_{e} S_{i} A v P_{i 2}}{4 S_{m}}
$$

In the expression, $u_{n i}$ is the noise ionic flow of detectable gas. Expression (16) shows that, only if the mass spectrometer work at a stable state, for specific leak detecting gas $i$, the leak rate is a function of accumulating time, leak gas pressure increment, sample gas increment and sample gas quantity, which means that comparing initial value, final value and sample value can ignore the instability and repetition of the QMS.

\section{TEST RESULT}

Employ the multi-system leak detecting system on spacecraft to test a new spacecraft. Choose inert gases which are little in air and have high critical pressure such as $\mathrm{He}\left(5 \times 10^{-6}\right), \mathrm{CF}_{4}\left(1 \times 10^{-6}\right)$, Ne $\left(1800 \times 10^{-6}\right), \mathrm{Kr}\left(1 \times 10^{-6}\right)$ and $\mathrm{SF}_{6}$ as detectable gases. Confirm mass number 4.00, 69.00, 22.00, $84.00,127.00$ as character peak value, adjust the pressure of mass spectrometer, SEM voltage, cathode voltage, focus voltage, field axis voltage, bias voltage and regulating voltage to make the QMS operating at the best state for the detectable gases. In the atmosphere, the minimal $\mathrm{He}$ concentration can be test is $13 \times 10^{-9}, \mathrm{CF}_{4}$ concentration is $10 \times 10^{-9}$, Ne concentration is $3 \times 10^{-}$ ${ }^{6}, \mathrm{SF}_{6}$ concentration is $0.3 \times 10^{-9}, \mathrm{Kr}$ concentration is $10 \times 10^{-9}$. For the collection chamber with $100 \mathrm{~m}^{3}$ volume, in 24 hours, the minimal He leak rate can be test is $1.5 \times 10^{-6} \mathrm{~Pa} \cdot \mathrm{m}^{3} / \mathrm{s}, \mathrm{CF}_{4}$ leak rate is $1.1 \times 10^{-6}$
$\mathrm{Pa} \cdot \mathrm{m}^{3} / \mathrm{s}$, Ne leak rate is $3.5 \times 10^{-5} \mathrm{~Pa} \cdot \mathrm{m}^{3} / \mathrm{s}, \mathrm{SF}_{6}$ leak rate is $3 \times 10^{-8} \mathrm{~Pa} \cdot \mathrm{m}^{3} / \mathrm{s}$, $\mathrm{Kr}$ leak rate is $1.1 \times 10^{-6}$ $\mathrm{Pa} \cdot \mathrm{m}^{3} / \mathrm{s}$.

\section{CONCLUSION}

The following conclusions can be drawn:

(1) Store the initial value gas by initial value container, which achieve that different detectable gases in different time period could be compared at same time, which can avoid the linearity and iteration problem of QMS.

(2) Increasing the diameter of sampling hole and increase the input sample would increase the ionic flow, but the pressure of mass spectrometer must be less than the upper limit of QMS working pressure and the detectable gas ionic flow must be twice bigger than noise ionic flow.

(3) Injection standard gas directly to calibrate multi-system leak detecting leak-rate, which avoid the calibrate problem of QMS. Controlling the quantity of Injecting to make the standard sample ionic flow and the final ionic flow the same dimension, which avoid the linearity of QMS to make the test more reliable.

(4) In atmosphere, for the collection chamber with $100 \mathrm{~m}^{3}$ volume, in 24 hours, the minimal leak rate can be test is $10^{-6} \mathrm{~Pa} \cdot \mathrm{m}^{3} / \mathrm{s}$, which accord with the total leak rate demand of spacecraft.

\section{REFERENCES}

[1] Sanger S. Leak detector for spacecraft testing with high accuracy. AIAA 812463.

[2] Sodal I E, Hanna D A. Improved mass spectrometer measurements using a pulsed gas sampling system. J. Vac. Sci. Technol. A 1997, 15 (1):196.

[3] Yan Rongxin. Research on multi-detector gas analysis machine. J Chinese Space Science and Technology. 1998, 18(6): 49.

[4] Yan Rongxin. The leak testing for spacecraft with quadrupole mass spectrometer. J Chinese Space Science and Technology2001, 21(4): 44.

[5] Rongxin Yan, The study of multi-system leak detection for spacecraft with quadrupole mass spectrometer, $20^{\text {th }}$ International Mass Spectrometry Conference, 2014, 08, 29, Geneva. Programe 44. 